SU-4252-756

May 2002

\title{
THE STAR PRODUCT ON THE FUZZY SUPERSPHERE
}

\author{
A. P. Balachandran, S. Kürkçüoğlu and E. Rojas 円 \\ Department of Physics, Syracuse University, Syracuse, NY 13244-1130, USA
}

\begin{abstract}
The fuzzy supersphere $S_{F}^{(2,2)}$ is a finite-dimensional matrix approximation to the supersphere $S^{(2,2)}$ incorporating supersymmetry exactly. Here the $\star$-product of functions on $S_{F}^{(2,2)}$ is obtained by utilizing the $\operatorname{OSp}(2,1)$ coherent states. We check its graded commutative limit to $S^{(2,2)}$ and extend it to fuzzy versions of sections of bundles using the methods of [1]. A brief discussion of the geometric structure of our $\star$-product completes our work.
\end{abstract}

\footnotetext{
${ }^{1}$ E-mails: bal@phy.syr.edu, skurkcuo@phy.syr.edu, erojas@phy.syr.edu
} 


\section{Introduction}

Studies of field theories on non-commutative (fuzzy) manifolds have started to produce many novel and encouraging results in the last few years. In these models one takes compact manifolds which are usually co-adjoint orbits of Lie groups and discretises them by quantization [2].

Upon quantization the discretized manifold exhibits a cell-like structure with the number of cells being finite. In this new non-commutative geometry without points, all symmetries of a field theory are generally preserved. With these properties, this recently divised technique could serve as a non-perturbative regulator for field theories [3, 4].

Among these manifolds, the fuzzy 2-sphere $S_{F}^{2}$ has been extensively studied. It has become evident that field theories on $S_{F}^{2}$ avoid fermion doubling as well as permit reformulation of sigma models and extended objects such as monopoles, instantons, etc [5, 6, 07.

An important ingredient in understanding the geometrical structure underlying these fuzzy models and their continuum limit is the associative $\star$-product of functions. Recently, explicit expressions for $\star$-product of functions on $S_{F}^{2}, C P_{F}^{N}$ (the fuzzy $C P_{N}$ ) and the fuzzy complex Grassmannian spaces have appeared in the literature [8, 9, 10]. One way that has been followed in these studies was first to introduce generalized Perelomov-like coherent states [11] and map an operator to a function on a fuzzy manifold by identifying its diagonal matrix elements with values of the function on the fuzzy manifold. It is a theorem that diagonal coherent state elements of operators completely determine that operator. An associative $\star$-product of two such functions is therefrom introduced straightforwardly and their properties are studied in detail. A finite series expansion of this product is obtained by the authors in terms of the derivatives of the functions involved and a projection operator enclosing the differential geometric structure of the manifold.

In this article we investigate the construction of an associative $\star$-product of functions on the fuzzy supersphere $S_{F}^{(2,2)}$. The latter have been studied in the articles by Grosse et al. [12, 14]. Our formulation of the problem will be based on [12] for the properties of the fuzzy $S_{F}^{(2,2)}$ and the work [9] for the introduction of the $\star$-product. Our construction of the coherent states on the supergroup $O S p(2,1)$ will rely on the use of annihilation-creation operators, however their equivalence to the $O S p(2,1)$ supergroup-induced coherent states will be explicitly shown. We also extend our results to obtain $\star$-products on "sections of bundles" on $S_{F}^{(2,2)}$ using the methods of [1].

Our text is organized as follows. In Section 2, to fix notation and conventions and to be self contained we briefly review the representation theory and basic properties of Lie superalgebras $\operatorname{osp}(2,1)$ and $\operatorname{ssp}(2,2)$ and the corresponding supergroups $O S p(2,1)$ and $O S p(2,2)$, which underlie the constrution of $S_{F}^{(2,2)}$. In Section 3, we take on the task of constructing the supercoherent states which will be used to induce the definition of the $\star$-product in a later section. Section 4 briefly summarizes the definition and properties of the usual and fuzzy superspheres $S^{(2,2)}$ and $S_{F}^{(2,2)}$ respectively from a group theoretic point of view. In Section 5, we introduce the $\star$-product on $S_{F}^{(2,2)}$ and compute it by utilizing the properties of supercoherent states of Section 3. The product and its properties are discussed in detail. This is followed by a discussion of (fuzzy) sections of bundles and the form of the $\star$-product for their elements. Section 6 includes remarks on the differential geometric structure underlying the $\star$-product. Some observations and discussion of further directions we are planning to explore in forthcoming studies conclude our work. 


\section{$2 \operatorname{osp}(2,1)$ and $\operatorname{osp}(2,2)$ Superalgebras and their Associated Su- pergroups}

Here we review some of the basic facts regarding the Lie superalgebras $\operatorname{osp}(2,1)$ and $\operatorname{osp}(2,2)$ and their associated supergroups $O S p(2,1)$ and $O S p(2,2)$. For detailed discussions, the reader is refered to the references 15, 16, 17, 18, 19].

\section{1 $\quad \operatorname{osp}(2,1)$ and $\operatorname{osp}(2,2)$ Superalgebras and their Representations}

Representations and properties of the Lie superalgebras have well-but not widely known features that we would like to briefly review for our purposes. As for any graded Lie algebra, osp $(2,1)$ and $\operatorname{ssp}(2,2)$ have even and odd parts. The even part of $\operatorname{osp}(2,1)$ is the Lie algebra $s u(2)$ with its usual generators $\Lambda_{i}(i, j=1,2,3)$. Its odd part is built up of $s u(2)$ spinors $\Lambda_{\alpha}(\alpha, \beta=4,5)$. They fulfill further properties to be explained below. The graded commutation relations are [17, 19]

$$
\begin{aligned}
{\left[\Lambda_{i}, \Lambda_{j}\right] } & =i \epsilon_{i j k} \Lambda_{k} \\
{\left[\Lambda_{i}, \Lambda_{\alpha}\right] } & =\frac{1}{2}\left(\sigma_{i}\right)_{\beta \alpha} \Lambda_{\beta}, \\
\left\{\Lambda_{\alpha}, \Lambda_{\beta}\right\} & =\frac{1}{2}\left(C \sigma_{i}\right)_{\alpha \beta} \Lambda_{i},
\end{aligned}
$$

where $\sigma_{i}$ are the Pauli matrices and $C_{\alpha \beta}=-C_{\beta \alpha}$ is the Levi-Civita symbol with $C_{45}=1$. (We use the indices 4,5 for their rows and columns.)

In the graded Lie algebras of our interest, the usual adjoint (or star) operation $\dagger$ on Lie algebras is replaced by the grade adjoint (or grade star) operation $\ddagger$ [18]. First, we note that the grade adjoint of an even (odd) element is even (odd). Next, one has $A^{\ddagger \ddagger}=(-1)^{|A|} A$ for an even or odd (that is homogeneous) element $A$ of degree $|A|(\bmod 2)$, or equally well, integer $(\bmod 2)$. (So, depending on $|A|,|A|$ itself can be taken 0 or 1.) Thus, it is the usual $\dagger$ on the even part, while on odd elements $A$, it squares to -1 . Furthermore, $[A, B\}^{\ddagger}=(-1)^{|A||B|}\left[B^{\ddagger}, A^{\ddagger}\right\}$ for homogeneous elements $A, B,[A, B]$ denoting the graded Lie bracket.

Henceforth we will denote the degree of $a$ (which may be a super Lie algebra element, a linear operator or an index $)$ by $|a|(\bmod 2),|a|$ denoting any integer in its equivalance class $<|a|+2 n$ : $n \in \mathbb{Z}>$.

Following 18, 19] we remark that any element of the $\operatorname{osp}(2,1)$ (and $\operatorname{osp}(2,2)$ ) graded Lie algebras has to fulfill certain "reality" properties implemented by $\ddagger$. For the generators of $\operatorname{osp}(2,1)$ these are given by

$$
\Lambda_{i}^{\ddagger}=\Lambda_{i}^{\dagger}=\Lambda_{i}, \quad \Lambda_{\alpha}^{\ddagger}=\sum_{\beta=4,5} C_{\alpha \beta} \Lambda_{\beta} \quad \alpha=4,5 .
$$

In a (grade star) representation of a graded Lie algebra on a graded vector space $V$, let $V=V_{0} \oplus V_{1}$ where $V_{0}$ and $V_{1}$ are even and odd subspaces [16]. $V_{0}$ and $V_{1}$ are invariant under the even elements of the graded Lie algebra while its odd elements map one to the other. Let us also assume that $V$ is endowed with the inner product $\langle u \mid v\rangle$ for all $u, v \in V$. Now if $L$ is a linear operator acting on $V$ then the grade adjoint of $L$ is defined by

$$
<L^{\ddagger} u\left|v>=(-1)^{|u||L|}<u\right| L v>.
$$


In a basis adapted to the above decomposition of $V, L$ has the matrix representation

$$
M_{L}=\left(\begin{array}{cc}
\alpha_{1} & \alpha_{2} \\
\alpha_{3} & \alpha_{4}
\end{array}\right)=M_{0}+M_{1} \quad M_{0}=\left(\begin{array}{cc}
\alpha_{1} & 0 \\
0 & \alpha_{4}
\end{array}\right), \quad M_{1}=\left(\begin{array}{cc}
0 & \alpha_{2} \\
\alpha_{3} & 0
\end{array}\right)
$$

where $M_{0}$ and $M_{1}$ are the even and odd parts of $M_{L}$. The formula for $\ddagger$ is then

$$
M_{L}^{\ddagger}=\left(\begin{array}{cc}
\alpha_{1}^{\dagger} & \alpha_{3}^{\dagger} \\
-\alpha_{2}^{\dagger} & \alpha_{4}^{\dagger}
\end{array}\right),
$$

$\alpha_{i}^{\dagger}$ being matrix adjoint of $\alpha_{i}$. We note that the supertrace $\operatorname{str}$ of $M_{L}$ is:

$$
\operatorname{str} M_{L}=\operatorname{Tr} \alpha_{1}-\operatorname{Tr} \alpha_{4} .
$$

The irreducible representations of $\operatorname{osp}(2,1)$ [12, 17, 19, 20] are characterized by an integer or half-integer non-negative quantum number $J_{o s p(2,1)}$ called superspin. From the point of view of the irreducible representations of $s u(2)$, the superspin $J_{o s p(2,1)}$ representation has the decomposition

$$
J_{o s p(2,1)}=J_{s u(2)} \oplus\left(J-\frac{1}{2}\right)_{s u(2)},
$$

where $J_{s u(2)}$ is the $s u(2)$ representation for angular momentum $J_{s u(2)}$. In particular the fundamental and adjoint representations of $\operatorname{osp}(2,1)$ correspond to $J_{o s p(2,1)}=1 / 2$ and $J_{o s p(2,1)}=1$ respectively, being 3 and 5 dimensional. The quadratic Casimir operator is given by

$$
K_{2}=\Lambda_{i} \Lambda_{i}+C_{\alpha \beta} \Lambda_{\alpha} \Lambda_{\beta} .
$$

It has the eigenvalues $J(J+1 / 2)$.

The $\operatorname{osp}(2,2)$ superalgebra 12, 19, 20] can be defined by introducing an even generator $\Lambda_{8}$ commuting with the $\Lambda_{i}$ and odd generators $\Lambda_{\alpha}$ with $\alpha=6,7$ in addition to the already existing ones for $\operatorname{osp}(2,1)$. The graded commutation relations for $\operatorname{osp}(2,2)$ are then

$$
\begin{aligned}
{\left[\Lambda_{i}, \Lambda_{j}\right] } & =i \epsilon_{i j k} \Lambda_{k} \\
{\left[\Lambda_{i}, \Lambda_{\alpha}\right] } & =\frac{1}{2}\left(\tilde{\sigma}_{i}\right)_{\beta \alpha} \Lambda_{\beta} \\
{\left[\Lambda_{i}, \Lambda_{8}\right] } & =0 \\
{\left[\Lambda_{8}, \Lambda_{\alpha}\right] } & =\tilde{\varepsilon}_{\alpha \beta} \Lambda_{\beta} \\
\left\{\Lambda_{\alpha}, \Lambda_{\beta}\right\} & =\frac{1}{2}\left(\tilde{C} \tilde{\sigma}_{i}\right)_{\alpha \beta} \Lambda_{i}+\frac{1}{4}(\tilde{\varepsilon} \tilde{C})_{\alpha \beta} \Lambda_{8}
\end{aligned}
$$

where $i, j=1,2,3$ and $\alpha, \beta=4,5,6,7$. In above we have used the matrices

$$
\tilde{\sigma}_{i}=\left(\begin{array}{cc}
\sigma_{i} & 0 \\
0 & \sigma_{i}
\end{array}\right) \quad \tilde{C}=\left(\begin{array}{cc}
C & 0 \\
0 & -C
\end{array}\right) \quad \tilde{\varepsilon}=\left(\begin{array}{cc}
0 & I_{2 \times 2} \\
I_{2 \times 2} & 0
\end{array}\right) .
$$

Their matrix elements are indexed by $4, \ldots, 7$.

In addition to (4), the new generators satisfy the "reality" conditions

$$
\Lambda_{\alpha}^{\ddagger}=-\sum_{\beta=6,7} \tilde{C}_{\alpha \beta} \Lambda_{\beta}, \quad \alpha=6,7, \quad \Lambda_{8}^{\ddagger}=\Lambda_{8}^{\dagger}=\Lambda_{8} .
$$


So we can write the $\operatorname{osp}(2,2)$ reality conditions for all $\alpha$ as $\Lambda_{\alpha}^{\ddagger}=\tilde{C}_{\alpha \beta} \Lambda_{\beta}$.

Irreducible representations of $\operatorname{osp}(2,2)$ fall into two categories, namely the typical and nontypical ones [12, 20]. Typical ones are reducible with respect to the $\operatorname{sp}(2,1)$ superalgebra (except for the trivial representation) whereas non-typical ones are irreducible. Typical representations are labeled by an integer or half integer non-negative number $J_{o s p(2,2)}$, called $\operatorname{osp}(2,2)$ superspin. These have the $\operatorname{ssp}(2,1)$ content $J_{o s p(2,2)}=J_{o s p(2,1)} \oplus(J-1 / 2)_{o s p(2,1)}$ for $J_{o s p(2,2)} \geq 1 / 2$ while $(0)_{o s p(2,2)}=(0)_{o s p(2,1)}$. Hence

$$
J_{o s p(2,2)}= \begin{cases}J_{s u(2)} \oplus\left(J-\frac{1}{2}\right)_{s u(2)} \oplus\left(J-\frac{1}{2}\right)_{s u(2)} \oplus(J-1)_{s u(2)}, & J_{o s p(2,2)} \geq 1 \\ \left(\frac{1}{2}\right)_{s u(2)}+(0)_{s u(2)}+(0)_{s u(2)}, & J_{o s p(2,2)}=\frac{1}{2}\end{cases}
$$

$o s p(2,2)$ has the quadratic Casimir operator

$$
\begin{aligned}
K_{2}^{\prime} & =\Lambda_{i} \Lambda_{i}+\tilde{C}_{\alpha \beta} \Lambda_{\alpha} \Lambda_{\beta}-\frac{1}{4} \Lambda_{8}^{2} \\
& =K_{2}-\left(\sum_{\alpha, \beta=6,7}-\tilde{C}_{\alpha \beta} \Lambda_{\alpha} \Lambda_{\beta}+\frac{1}{4} \Lambda_{8}^{2}\right) .
\end{aligned}
$$

Note that since all the generators of $\operatorname{osp}(2,1)$ commute with $K_{2}^{\prime}$ and $K_{2}$, they also commute with

$$
V:=-\sum_{\alpha, \beta=6,7} \tilde{C}_{\alpha \beta} \Lambda_{\alpha} \Lambda_{\beta}+\frac{1}{4} \Lambda_{8}^{2} .
$$

As regards the non-typical representations of $\operatorname{osp}(2,2)$ associated with $J_{o s p(2,1)}$, we note that the substitutions

$$
\Lambda_{i} \rightarrow \Lambda_{i}, \quad \Lambda_{\alpha} \rightarrow \Lambda_{\alpha}, \quad \alpha=4,5 ; \quad \Lambda_{\alpha} \rightarrow-\Lambda_{\alpha}, \quad \alpha=6,7 ; \quad \Lambda_{8} \rightarrow-\Lambda_{8}
$$

define an automorphism of $\operatorname{osp}(2,2)$. This automorphism changes a non-typical irreducible representation into an inequivalent one (except for the trivial representation with $J=0$ ), while preserving the reality conditions given in (41) and (17) [19]. We discriminate between these two representations associated with $J_{o s p(2,1)}$ as follows: For $J>0, \hat{J}_{o s p(2,2)+}$ will denote the representation in which the eigenvalue of the representative of $\Lambda_{8}$ on vectors with angular momentum $J$ is positive and $\hat{J}_{o s p(2,2)-}$ will denote its partner where this eigenvalue is negative. (This eigenvalue is zero only in the trivial representation with $J=0$.) In this paper we concentrate on $\hat{J}_{o s p(2,2)+}$. The results for $\hat{J}_{\operatorname{ssp}(2,2)-}$ are similar and will be occasionally indicated.

Below we list some of the well-known results and standard notations that are used throughout the text 17, 19, 21]. The fundamental representation of $\operatorname{osp}(2,2)$ is non-typical and we concentrate on the one given by $\hat{J}_{o s p(2,2)+}=\left(\frac{\hat{1}}{2}\right)_{o s p(2,2)+}$. It is generated by the $(3 \times 3)$ supertraceless matrices $\Lambda_{a}^{\left(\frac{1}{2}\right)}$ satisfying the "reality" conditions of (41) and (17):

$$
\begin{array}{rlrl}
\Lambda_{i}^{\left(\frac{1}{2}\right)}=\frac{1}{2}\left(\begin{array}{cc}
\sigma_{i} & 0 \\
0 & 0
\end{array}\right) & \Lambda_{4}^{\left(\frac{1}{2}\right)}=\frac{1}{2}\left(\begin{array}{cc}
0 & \xi \\
\eta^{T} & 0
\end{array}\right) \quad \Lambda_{5}^{\left(\frac{1}{2}\right)}=\frac{1}{2}\left(\begin{array}{cc}
0 & \eta \\
-\xi^{T} & 0
\end{array}\right) \\
\Lambda_{6}^{\left(\frac{1}{2}\right)}=\frac{1}{2}\left(\begin{array}{cc}
0 & -\xi \\
\eta^{T} & 0
\end{array}\right) & \Lambda_{7}^{\left(\frac{1}{2}\right)}=\frac{1}{2}\left(\begin{array}{cc}
0 & -\eta \\
-\xi^{T} & 0
\end{array}\right) & \Lambda_{8}^{\left(\frac{1}{2}\right)}=\left(\begin{array}{cc}
I_{2 \times 2} & 0 \\
0 & 2
\end{array}\right)
\end{array}
$$


where

$$
\xi=\left(\begin{array}{c}
-1 \\
0
\end{array}\right) \quad \text { and } \quad \eta=\left(\begin{array}{c}
0 \\
-1
\end{array}\right)
$$

These generators satisfy

$$
\Lambda_{a}^{\left(\frac{1}{2}\right)} \Lambda_{b}^{\left(\frac{1}{2}\right)}=S_{a b} \mathbf{1}+\frac{1}{2}\left(d_{a b c}+i f_{a b c}\right) \Lambda_{c}^{\left(\frac{1}{2}\right)} \quad(a, b, c=1,2, \ldots 8)
$$

with

$$
\begin{aligned}
S_{a b} & =\operatorname{str}\left(\Lambda_{a}^{\left(\frac{1}{2}\right)} \Lambda_{b}^{\left(\frac{1}{2}\right)}\right), \\
f_{a b c} & =\operatorname{str}\left(-i\left[\Lambda_{a}^{\left(\frac{1}{2}\right)}, \Lambda_{b}^{\left(\frac{1}{2}\right)}\right\} \Lambda_{c}^{\left(\frac{1}{2}\right)}\right), \\
d_{a b c} & =\operatorname{str}\left(\left\{\Lambda_{a}^{\left(\frac{1}{2}\right)}, \Lambda_{b}^{\left(\frac{1}{2}\right)}\right] \Lambda_{c}^{\left(\frac{1}{2}\right)}\right) .
\end{aligned}
$$

Here $a=i=1,2,3$, and $a=8$ label the even generators and $a=\alpha=4,5,6,7$ label the odd generators. Also here and what follows, $[A, B\},\{A, B]$ denote the graded commutator and anticommutator respectively: If $A$ and $B$ are homogeneous elements, $[A, B\}=A B-(-1)^{|A||B|} B A,\{A, B]=$ $A B+(-1)^{|A||B|} B A$.

$S_{a b}$ defines the invariant metric of the super Lie algebra $\operatorname{osp}(2,2)$. In their block diagonal form, $S$ and its inverse read

$$
S=\left(\begin{array}{ccc}
\frac{1}{2} I & & \\
& -\frac{1}{2} \tilde{C} & \\
& & -2
\end{array}\right)_{8 \times 8} \quad S^{-1}=\left(\begin{array}{ccc}
2 I & & \\
& 2 \tilde{C} & \\
& & -\frac{1}{2}
\end{array}\right)_{8 \times 8} .
$$

The explicit values of the structure constants $f_{a b c}$ can be read from (16), since $\left[\Lambda_{a}, \Lambda_{b}\right\}=i f_{a b c} \Lambda_{c}$. Those of $d_{a b c}$ are as follows 円:

$$
\begin{aligned}
& d_{i j 8}=-\frac{1}{2} \delta_{i j}, \quad d_{\alpha \beta 8}=\frac{3}{4} \tilde{C}_{\alpha \beta}, \quad d_{\alpha 8 \beta}=3 \delta_{\alpha \beta}, \quad d_{i 8 j}=2 \delta_{i j}, \\
& d_{\alpha \beta i}=-\frac{1}{2}\left(\tilde{\varepsilon} \tilde{C} \tilde{\sigma}_{i}\right)_{\alpha \beta}, \quad d_{i \alpha \beta}=-\frac{1}{2}\left(\tilde{\varepsilon} \tilde{\sigma}_{i}\right)_{\beta \alpha}, \quad d_{888}=6 .
\end{aligned}
$$

We close this subsection with a final remark. Discussion in the subsequent sections will involve the use of linear operators acting on the adjoint representation of $\operatorname{sep}(2,2)$. These are linear operators $\hat{\mathcal{Q}}$ acting on $\Lambda_{a}$ according to $\hat{\mathcal{Q}} \Lambda_{a}=\Lambda_{b} \mathcal{Q} b a, \mathcal{Q}$ being the matrix representation of $\hat{\mathcal{Q}}$. They are graded because $\Lambda_{a}$ 's are, and hence the linear operators on the adjoint representation are graded. The degree (or grade) of a matrix $\mathcal{Q}$ with only the nonzero entry $\mathcal{Q}_{a b}$ is $\left(\left|\Lambda_{a}\right|+\right.$ $\left.\left|\Lambda_{b}\right|\right)(\bmod 2) \equiv(|a|+|b|)(\bmod 2)$. The grade star operation on $\hat{\mathcal{Q}}$ now follows from the sesquilinear form

$$
\left(\alpha=\alpha_{a} \Lambda_{A}, \beta=\beta_{b} \Lambda_{b}\right)=\bar{\alpha}_{a} S_{a b} \beta_{b}, \quad \alpha_{a}, \beta_{b} \in \mathbb{C}
$$

and is given by

$$
\left(\hat{\mathcal{Q}}^{\ddagger} \alpha, \beta\right)=(-1)^{|\alpha||\hat{\mathcal{Q}}|}(\alpha, \hat{\mathcal{Q}} \beta) .
$$

\footnotetext{
${ }^{1}$ The tensor $d_{a b c}$ given explicitly in (27) for $\hat{J}_{o s p(2,2)+}$ becomes $-d_{a b c}$ for $\hat{J}_{o s p(2,2)-}$.
} 


\section{$2.2 \quad$ Passage to Supergroups}

We recollect here the passage from these superalgebras to the corresponding supergroups [16, 21]. Let $\xi_{a},(a=1, \ldots, 8)$ be the super coordinates. Here $a=i=1,2,3$ and $a=8$ label the even and $a=\alpha=4,5,6,7$ label the odd coordinates. $\xi_{a}$ satisfy the graded commutation relations mutually and with the algebra elements:

$$
\left[\xi_{a}, \xi_{b}\right\}=0, \quad\left[\xi_{a}, \Lambda_{b}\right\}=0
$$

We assume that $\xi_{i}^{\ddagger}=\xi_{i}, \xi_{8}^{\ddagger}=\xi_{8}$ and $\xi_{\alpha}^{\ddagger}=\tilde{C}_{\alpha \beta} \xi_{\beta}$. Then $\xi_{a} \Lambda_{a}$ is grade star even:

$$
\left(\xi_{a} \Lambda_{a}\right)^{\ddagger}=\xi_{a} \Lambda_{a} .
$$

(31) corresponds to the usual hermiticity property of Lie algebras which yields unitary representations of the group. The supergroup elements $g$ are $e^{i \xi_{a} \Lambda_{a}}$ and products of such elements. Note that $g^{\ddagger}=g^{-1}$.

\section{Coherent States}

In this section we construct the supercoherent states (SCS) that are appropriate for our purposes. Alternative approaches for constructing $\operatorname{OSp}(2,1)$ coherent states are given in the literature [21, 22]. The SCS constructed here will be used heavily in the following sections.

We start our discussion by introducing the coherent state including the bosonic and fermionic degrees of freedom [11, 23]:

$$
\begin{gathered}
|\psi>\equiv| z, \theta>=\frac{e^{-1 / 2|\psi|^{2}}}{|\psi|} e^{a_{\alpha}^{\dagger} z_{\alpha}+b^{\dagger} \theta} \mid 0> \\
|\psi|^{2} \equiv\left|z_{1}\right|^{2}+\left|z_{2}\right|^{2}+\bar{\theta} \theta
\end{gathered}
$$

Here $a_{\alpha}, a_{\alpha}^{\dagger}(\alpha=1,2), b, b^{\dagger}$ are bosonic and fermionic annihilation and creation operators with the usual commutation and anticommutation relations

$$
\left[a_{\alpha}, a_{\beta}^{\dagger}\right]=\delta_{\alpha \beta}, \quad\left\{b, b^{\dagger}\right\}=1, \quad\left[a_{\alpha}, b\right]=0
$$

etc. $\theta$ is a Grassmann number such that $\{\theta, \bar{\theta}\}=0$ and $\theta \theta=\bar{\theta} \bar{\theta}=0$. We have also fixed the normalization of $\mid \psi>$ 's:

$$
<\psi \mid \psi>=\frac{1}{|\psi|^{2}}
$$

In order to define our supercoherent states we require a finite-dimensional setting. For this purpose we will project the state in (32) to the $N$-dimensional Fock space. The projection operator is given by

$$
P_{N}=\sum_{N=n_{1}+n_{2}+n_{3}} \frac{1}{n_{1} ! n_{2} !}\left(a_{1}^{\dagger}\right)^{n_{1}}\left(a_{2}^{\dagger}\right)^{n_{2}}\left(b^{\dagger}\right)^{n_{3}}|0><0|(b)^{n_{3}}\left(a_{2}\right)^{n_{2}}\left(a_{1}\right)^{n_{1}} .
$$


Note that $n_{3}=0$ or 1 and that $P_{N}^{2}=P_{N}, P_{N}^{\dagger}=P_{N}$.

Projecting $|\psi\rangle$ with $P_{N}$ to the $N$-dimensional Fock space and renormalizing the result by the factor $\left.\left(<\psi\left|P_{N}\right| \psi\right\rangle\right)^{1 / 2}$, we get

$$
\left|\psi, N>=\frac{1}{\sqrt{N !}} \frac{\left(a_{\alpha}^{\dagger} z_{\alpha}+b^{\dagger} \theta\right)^{N}}{(|\psi|)^{N}}\right| 0>.
$$

This is exactly the supercoherent state we are looking for. It can be derived in another way. Consider the following highest weight state in the $J_{o s p(2,1)}=1 / 2$ representation of $\operatorname{osp}(2,1)$ for which $N=1$ :

$$
\left|J, J_{3}>=\right| 1 / 2,1 / 2>\text {. }
$$

This is also the highest weight state in the associated non-typical representation $\hat{J}_{\text {osp }(2,2)+}=$ $\left(\frac{\hat{1}}{2}\right)_{o s p(2,2)+}$ of $\operatorname{osp}(2,2)$. Consider now the action of the $O S p(2,1)$ group on (38). This can be realized by taking $g \in O S p(2,1)$ and $\mathcal{U}(g)$ as the corresponding element in the $3 \times 3$ fundamental representation. Thus let

$$
|g>=\mathcal{U}(g)| 1 / 2,1 / 2>,
$$

where $\mid g>$ is the super analogue of the Perelomov coherent state [21]. Write

$$
\left|1 / 2,1 / 2>=\Psi_{1}^{\dagger}\right| 0>
$$

where

$$
\Psi^{\dagger}=\left(\Psi_{1}^{\dagger}, \Psi_{2}^{\dagger}, \Psi_{0}^{\dagger}\right) \equiv\left(a_{1}^{\dagger}, a_{2}^{\dagger}, b^{\dagger}\right)
$$

Let also 21]

$$
\mathcal{D}(g)=\left(\begin{array}{ccc}
z_{1}^{\prime} & -\bar{z}_{2}^{\prime} & -\chi \\
z_{2}^{\prime} & \bar{z}_{1}^{\prime} & -\bar{\chi} \\
\theta^{\prime} & -\bar{\theta}^{\prime} & \lambda
\end{array}\right), \quad \sum_{i}\left|z_{i}^{\prime}\right|^{2}+\bar{\theta}^{\prime} \theta^{\prime}=1
$$

be the form of the matrix of $\mathcal{U}(g)$ in the basis $\left\{\Psi_{\mu}^{\dagger} \mid 0>\right\}(\mu=1,2,0)$. Then

$$
\begin{aligned}
\mid g> & =\Psi_{\mu}^{\dagger}(\mathcal{D}(g))_{\mu 1} \mid 0> \\
& =\left(a_{\alpha}^{\dagger} z_{\alpha}^{\prime}+b^{\dagger} \theta^{\prime}\right)\left|0>=\Psi_{\mu}^{\dagger} \psi_{\mu}^{\prime}\right| 0>,
\end{aligned}
$$

with

$$
\psi^{\prime}=\left(\begin{array}{c}
\psi_{1}^{\prime} \\
\psi_{2}^{\prime} \\
\psi_{0}^{\prime}
\end{array}\right) \equiv\left(\begin{array}{c}
z_{1}^{\prime} \\
z_{2}^{\prime} \\
\theta^{\prime}
\end{array}\right) .
$$

(43) is exactly equal to $\mid \psi, 1>$ in (37) if we make the identification

$$
\psi_{\mu}^{\prime}=\frac{\psi_{\mu}}{|\psi|} .
$$


For the case of general $N$ we start from the highest weight state $\mid N / 2, N / 2>$ in the $N$-fold graded symmetric tensor product $\otimes_{G}^{N}$ of the $J_{o s p(2,1)}=1 / 2$ representation and the corresponding representative $\mathcal{U}_{G}^{\otimes}(g)$ of $g$ :

$$
\begin{aligned}
\mid N / 2, N / 2> & :=\left|1 / 2,1 / 2>\otimes_{G} \cdots \cdots \otimes_{G}\right| 1 / 2,1 / 2>, \\
\mathcal{U}^{\otimes_{G}^{N}}(g) & :=\mathcal{U}(g) \otimes_{G} \cdots \cdots \otimes_{G} \mathcal{U}(g) .
\end{aligned}
$$

Note that, since $\mathcal{U}(g)$ is an element of $\operatorname{OSp}(2,1)$, it is even. The corresponding coherent state is

$$
\left|g ; N / 2>=\mathcal{U}_{G}^{\otimes_{G}}\right| N / 2, N / 2>=\mathcal{U}(g)\left|1 / 2,1 / 2>\otimes_{G} \cdots \cdots \otimes_{G} \mathcal{U}(g)\right| 1 / 2,1 / 2>.
$$

Upon using (43) and (45), this becomes equivalent to (37).

\section{On the Superspheres}

\subsection{The Commutative Supersphere $S^{(2,2)}$}

There exists an important map from the supercoherent states to orbits in the adjoint representation of $O S p(2,1)$ and $O S p(2,2)$. The former is the supersphere [24, 25, 26]

$$
S^{(2,2)}=O S p(2,1) / U(1)
$$

while the latter is a closely related orbit.

We first observe that the $\operatorname{osp}(2,1)$ generators $\Lambda_{a}(a \leq 5)$ in the super Fock space are represented by

$$
\lambda_{a}=\Psi_{\mu}^{\dagger}\left(\Lambda_{a}^{\left(\frac{1}{2}\right)}\right)_{\mu \nu} \Psi_{\nu}
$$

The supergroup action preserves $|\psi|^{2}$. So consider the following map $\pi$ from the $(3,2)$ dimensional supersphere $S^{(3,2)}=<\psi:|\psi|^{2}=$ constant $\neq 0>$ to functions on $S^{(2,2)}$ :

$$
\begin{gathered}
\pi: \quad \psi \longrightarrow<\psi, 1\left|\lambda_{a}\right| \psi, 1>:=\phi_{a}(\psi, \bar{\psi}), \\
\phi_{a}(\psi, \bar{\psi})=\frac{1}{|\psi|^{2}} \bar{\psi} \Lambda_{a}^{\left(\frac{1}{2}\right)} \psi
\end{gathered}
$$

The overall phase of $\psi$ cancels out while no other degree of freedom is lost on R.H.S. For this reason, this is a map from $S^{(3,2)}$ to the $(2,2)$ dimensional space 2

$$
S^{(2,2)}:=S^{(3,2)} / U(1)=\left\{\phi(\psi)=\left(\phi_{1}(\psi), \cdots, \phi_{5}(\psi)\right)\right\} .
$$

$\pi$ is thus the "super Hopf Fibration" over $S^{(2,2)}$ [24, 25], the "super" generalization of the Hopf fibration $U(1) \rightarrow S^{3} \rightarrow S^{2}$. $S^{(2,2)}$ can be thought as the supersphere generalizing $S^{2}$.

We now characterize $S^{(2,2)}$ as an adjoint orbit of $O S p(2,1)$. First observe that $\phi(\psi)$ is a (super)vector in the adjoint representation of $O S p(2,1)$. Under the action

$$
\phi \rightarrow g \phi, \quad(g \phi)(\psi)=\phi\left(g^{-1} \psi\right), \quad g \in O S p(2,1)
$$

\footnotetext{
${ }^{2}$ In what follows we do not show the $\bar{\psi}$ depedence of $\phi_{a}$ to abbreviate the notation a little bit.
} 
it transforms by the adjoint representation $g \rightarrow A d g$ :

$$
\phi_{a}\left(g^{-1} \psi\right)=\phi_{b}(\psi)(A d g)_{b a} .
$$

The generators of $\operatorname{osp}(2,1)$ in the adjoint representation are $a d \Lambda_{a}$ where

$$
\left(a d \Lambda_{a}\right)_{c b}=i f_{a b c} .
$$

From this and the infinitesimal variations $\delta \phi(\psi)=\varepsilon_{a} a d \Lambda_{a} \phi(\psi)$ of $\phi(\psi)$ under the adjoint action with $\varepsilon_{i}$ even and $\varepsilon_{\alpha}$ odd Grassmann variables, we can verify that

$$
\delta\left(\phi_{i}(\psi)^{2}+C_{\alpha \beta} \phi_{\alpha}(\psi) \phi_{\beta}(\psi)\right)=0 .
$$

Hence, $S^{(2,2)}$ is an $O S p(2,1)$ orbit with the invariant

$$
\phi_{i}(\psi)^{2}+C_{\alpha \beta} \phi_{\alpha}(\psi) \phi_{\beta}(\psi) \text {. }
$$

The value of the invariant can of course be changed by scaling. Now the even components of $\phi_{a}(\psi)$ are real while its odd entries depend on both $\theta$ and $\bar{\theta}$ :

$$
\phi_{i}(\psi)=\frac{1}{2} \frac{1}{|\psi|^{2}} \bar{z} \sigma_{i} z, \quad \phi_{4}(\psi)=-\frac{1}{2} \frac{1}{|\psi|^{2}}\left(\bar{z}_{1} \theta+z_{2} \bar{\theta}\right), \quad \phi_{5}(\psi)=\frac{1}{2} \frac{1}{|\psi|^{2}}\left(-\bar{z}_{2} \theta+z_{1} \bar{\theta}\right) .
$$

Let us define $\ddagger$ to be the usual adjoint operation $\dagger$ on $\Psi_{\mu}$ and $\Psi_{\mu}^{\dagger}$. Then by the requirements that

$$
\begin{aligned}
<\psi, 1\left|\lambda_{a}\right| \psi, 1>^{\ddagger}= & <\psi, 1\left|\lambda_{a}^{\ddagger}\right| \psi, 1>, \\
\lambda_{i}^{\ddagger}=\lambda_{i} & , \quad \lambda_{\alpha}^{\ddagger}=C_{\alpha \beta} \lambda_{\beta},
\end{aligned}
$$

one deduces that

$$
\phi_{i}(\psi)^{\ddagger}=\phi_{i}(\psi) \quad \phi_{\alpha}(\psi)^{\ddagger}=C_{\alpha \beta} \phi_{\beta}(\psi),
$$

and that

$$
z_{i}^{\ddagger}=\bar{z}_{i}, \quad \bar{z}_{i}^{\ddagger}=z_{i}, \quad \theta^{\ddagger}=-\bar{\theta}, \quad \bar{\theta}^{\ddagger}=\theta .
$$

(61) preserves the condition $|\psi|^{2}=$ constant and the $\operatorname{OSp}(2,1)$ orbit. The reality condition (60) reduces the degrees of freedom in $\phi_{\alpha}(\psi)$ to two. The $(3,2)$ variables $\phi_{a}(\psi)$ are further reduced to $(2,2)$ on fixing the value of the invariant (57). As $(2,2)$ is the dimension of $S^{(2,2)}$, there remains no further invariant in this orbit. Thus

$$
S^{(2,2)}=<\eta^{(+)} \in \mathbb{C}^{(3,2)}:\left(\eta_{i}^{(+)}\right)^{2}+C_{\alpha \beta} \eta_{\alpha}^{(+)} \eta_{\beta}^{(+)}=\frac{1}{4},\left(\eta_{i}^{(+)}\right)^{\ddagger}=\eta_{i}^{(+)},\left(\eta_{\alpha}^{(+)}\right)^{\ddagger}=C_{\alpha \beta} \eta_{\beta}^{(+)}>
$$

where we chose $\frac{1}{4}$ for the value of the invariant.

As $O S p(2,2)$ acts on $\psi$, that is on $S^{(3,2)}$, preserving the $U(1)$ fibres in the map $S^{(3,2)} \rightarrow S^{(2,2)}$, it has an action on the latter. It is not the adjoint action, but closely related to it, as we now explain. 
The nature of the $O S p(2,2)$ action on $S^{(2,2)}$ has elements of subtlety. If $g \in O S p(2,2)$ and $\psi \in S^{(3,2)}$ then $g \psi \in S^{(3,2)}$ and hence $\phi(g \psi) \in S^{(2,2)}$ :

$$
\begin{gathered}
\phi_{i}(g \psi)^{2}+C_{\alpha \beta} \phi_{\alpha}(g \psi) \phi_{\beta}(g \psi)=\frac{1}{4}, \\
\phi_{\alpha}^{\ddagger}(g \psi)=C_{\alpha \beta} \phi_{\beta}(g \psi) .
\end{gathered}
$$

But the expansion of $\phi_{\alpha}(g \psi)$ for infinitesimal $g$ contains not only the even Majorana spinors $\eta_{\alpha}^{(+)}$, but also the odd ones $\eta_{\alpha}^{(-)}$, where $\left(\eta_{\alpha}^{(-)}\right)^{\ddagger}=\sum_{\beta=6,7} \tilde{C}_{\alpha \beta} \eta_{\beta}^{(-)}(\alpha=6,7)$. We cannot thus think of the $O S p(2,2)$ action as an adjoint action on the adjoint space of $O S p(2,1)$. The reason of course is that the super Lie algebra $\operatorname{osp}(2,1)$ is not invariant under graded commutation with the generators $\Lambda_{6,7,8}$ of $\operatorname{osp}(2,2)$.

Now consider the generalization of the map (50) to the $\operatorname{sep}(2,2)$ Lie algebra,

$$
\psi \rightarrow<\psi, 1\left|\lambda_{a}\right| \psi, 1>:=\Phi_{a}(\psi), \quad a=1, \ldots, 8
$$

where $\lambda_{a}$ is given by the formula (49) for all $a$ and the $\bar{\psi}$ dependence of $\Phi_{a}$ has not been shown. Just as for $O S p(2,1)$, we find,

$$
\Phi_{a}\left(g^{-1} \psi\right)=\Phi_{b}(\psi)(A d g)_{b a}, \quad a, b=1, \ldots, 8, \quad g \in O S p(2,2) .
$$

Thus this extended vector $\Phi(\psi)=\left(\Phi_{1}(\psi), \Phi_{2}(\psi), \ldots, \Phi_{8}(\psi)\right)$ transforms as an adjoint (super)vector of $\operatorname{ssp}(2,2)$ under $O S p(2,2)$ action. The formula given in (51) extends to this case where index $a$ there also takes the values $(6,7,8)$. Note that with

$$
\begin{aligned}
& \Phi_{6}(\psi)=\frac{1}{2} \frac{1}{|\psi|^{2}}\left(\bar{z}_{1} \theta-z_{2} \bar{\theta}\right), \quad \Phi_{7}(\psi)=\frac{1}{2} \frac{1}{|\psi|^{2}}\left(\bar{z}_{2} \theta+z_{1} \bar{\theta}\right), \\
& \Phi_{8}(\psi)=\frac{1}{|\psi|^{2}}\left(\bar{z}_{i} z_{i}+2 \bar{\theta} \theta\right)=\left(2-\frac{1}{|\psi|^{2}} \bar{z}_{i} z_{i}\right)
\end{aligned}
$$

we have

$$
\Phi_{8}(\psi)^{\ddagger}=\Phi_{8}(\psi) \quad \Phi_{\alpha}(\psi)^{\ddagger}=\sum_{\beta=6,7} \tilde{C}_{\alpha \beta} \Phi_{\beta}(\psi), \quad \alpha=6,7
$$

showing that the new spinor $\Phi_{\alpha}(\psi),(\alpha=6,7)$ is an odd Majorana spinor as previous remarks suggested.

As $\Phi(\psi)$ transforms as an adjoint vector under $\operatorname{OSp}(2,2)$, the $O S p(2,2)$ Casimir function evaluated at $\Phi(\psi)$ is a constant on this orbit:

$$
\Phi_{i}^{2}(\psi)+\tilde{C}_{\alpha \beta} \Phi_{\alpha}(\psi) \Phi_{\beta}(\psi)-\frac{1}{4} \Phi_{8}^{2}(\psi)=\text { constant }
$$

But we saw that the sum of the first term, and the second term with $\alpha, \beta=4,5$ only, is invariant under $O S p(2,2)$. Hence so are the remaining terms:

$$
\sum_{\alpha, \beta=6,7} \tilde{C}_{\alpha \beta} \Phi_{\alpha}(\psi) \Phi_{\beta}(\psi)-\frac{1}{4} \Phi_{8}(\psi)^{2}=\text { constant } .
$$


Its value is $-\frac{1}{4}$ as can be calculated by setting $\psi=(1,0,0)$.

In fact since the $O S p(2,1)$ orbit has the dimension of $S^{(3,2)} / U(1)$ and $\Phi_{a}(\psi)=\Phi_{a}\left(\psi e^{i \gamma}\right)$ are functions of this orbit, we can completely express the latter in terms of $\phi(\psi)$. We find

$$
\begin{aligned}
& \Phi_{6}(\psi)=-2\left(\phi_{3}(\psi) \phi_{4}(\psi)+\left(\phi_{1}(\psi)+i \phi_{2}(\psi)\right) \phi_{5}(\psi)\right), \\
& \Phi_{7}(\psi)=2\left(\phi_{3}(\psi) \phi_{5}(\psi)-\left(\phi_{1}(\psi)-i \phi_{2}(\psi)\right) \phi_{4}(\psi)\right), \\
& \Phi_{8}(\psi)=2\left(1-\sqrt{\phi_{i}(\psi)^{2}}\right) .
\end{aligned}
$$

The generalization of the above arguments will consider the $N$-particle sector and the map

$$
\psi \rightarrow<\psi, N\left|\lambda_{a}\right| \psi, N>
$$

but that brings in nothing new, R.H.S. being $N \Phi_{a}(\psi)$.

\subsection{The Noncommutative (Fuzzy) Supersphere $S_{F}^{(2,2)}$}

The fuzzy supersphere $S_{F}^{(2,2)}$ is obtained by replacing $\Lambda_{a}$ with the scaled $\operatorname{osp}(2,1)$ generators $\Lambda_{a}(J)=\frac{\Lambda_{a}}{\sqrt{2} \sqrt{J\left(J+\frac{1}{2}\right)}}(a \leq 5)$. The relations that describe $S_{F}^{(2,2)}$ are then

$$
\begin{gathered}
\Lambda_{i}(J) \Lambda_{i}(J)+C_{\alpha \beta} \Lambda_{\alpha}(J) \Lambda_{\beta}(J)=\frac{1}{2}, \\
{\left[\Lambda_{a}(J), \Lambda_{b}(J)\right\}=\frac{1}{\sqrt{2} \sqrt{J\left(J+\frac{1}{2}\right)}} i f_{a b c} \Lambda_{c}(J),}
\end{gathered}
$$

the left-hand side of (73) being proportional to the Casimir operator $K_{2}$. The first of these two equations describes a supersphere of radius $\frac{1}{\sqrt{2}}$, parametrized by the matrices $\Lambda_{a}(J)$ whereas the second one measures the amount of noncommutativity between any two $\Lambda_{a}(J)$.

The graded commutative limit is recovered when $J \rightarrow \infty,\left[\Lambda_{a}(\infty), \Lambda_{b}(\infty)\right\} \rightarrow 0$. The graded commutator in (74) naturally extends to the $\operatorname{osp}(2,2)$ algebra $(a \leq 8)$, making the supersymmetry richer and allowing us to compute $\star$-products on $S_{F}^{(2,2)}$ as we now show.

\section{The $\star$-Products}

\section{$5.1 \quad$-Product on $S_{F}^{(2,2)}$}

First we remark that an operator (under suitable conditions) is completely determined by its diagonal matrix elements between standard coherent states [23]. This is also true for diagonal matrix elements between the supercoherent states (37). The diagonal elements of an operator for the supercoherent states (37) defines a function on $S_{F}^{(2,2)}$. We can define a $\star$-product on functions using this map from operators to functions as we shall see below.

We first determine the above map from operators to functions. It is sufficient to compute the matrix elements of the coordinate operators $\lambda_{a}$, the generalization to arbitrary operators can then

\footnotetext{
${ }^{3} \Phi_{6,7,8}$ become $-\Phi_{6,7,8}$ for $\hat{J}_{o s p(2,2)-}$.
} 
be made easily. One can write $\lambda_{a}$ as in (49). Proceding straightforwardly, the diagonal coherent state matrix element for $\lambda_{a}$,

$$
\begin{aligned}
W_{a}(\psi, \bar{\psi}, N) & =W_{a}\left(z_{1}, z_{2}, \bar{z}_{1}, \bar{z}_{2}, \theta, \bar{\theta}\right) \\
& =<\psi, N\left|\lambda_{a}\right| \psi, N>
\end{aligned}
$$

can be calculated to be

$$
W_{a}(\psi, \bar{\psi}, N)=\frac{1}{|\psi|^{2}} N \bar{\psi} \Lambda_{a}^{\left(\frac{1}{2}\right)} \psi
$$

To remove the $N$ dependence in $(76)$, we let

$$
\mathcal{W}_{a}\left(\psi^{\prime}, \bar{\psi}^{\prime}\right)=\frac{1}{N} W_{a}\left(\psi^{\prime}, \bar{\psi}^{\prime}, N\right)=\bar{\psi}^{\prime} \Lambda_{a}^{\left(\frac{1}{2}\right)} \psi^{\prime}
$$

where $\psi^{\prime}=\frac{\psi}{|\psi|}$ and $\mathcal{W}_{a}$ is a superfunction of $\psi^{\prime}, \bar{\psi}^{\prime}$.

We are now ready to define and compute the star product of two functions of the form $\mathcal{W}_{a}$ and $\mathcal{W}_{b}$. It depends on $N$, so we denote it by $\star_{N}$. It is given by [8, 9]

$$
\mathcal{W}_{a} \star_{N} \mathcal{W}_{b}\left(\psi^{\prime}, \bar{\psi}^{\prime}, N\right)=\frac{1}{N^{2}}<\psi^{\prime}, N\left|\lambda_{a} \lambda_{b}\right| \psi^{\prime}, N>
$$

which becomes, after a little manipulation

$$
\mathcal{W}_{a} \star_{N} \mathcal{W}_{b}\left(\psi^{\prime}, \bar{\psi}^{\prime}, N\right)=\frac{1}{N} \bar{\psi}^{\prime}\left(\Lambda_{a}^{\left(\frac{1}{2}\right)} \Lambda_{b}^{\left(\frac{1}{2}\right)}\right) \psi^{\prime}+\frac{N-1}{N}\left(\bar{\psi}^{\prime} \Lambda_{a}^{\left(\frac{1}{2}\right)} \psi^{\prime}\right)\left(\bar{\psi}^{\prime} \Lambda_{b}^{\left(\frac{1}{2}\right)} \psi^{\prime}\right) .
$$

Furthermore, since $\psi^{\prime} \Lambda_{a} \Lambda_{b} \psi^{\prime}$ is $\mathcal{W}_{a} \star_{1} \mathcal{W}_{b}$, this can be rewritten as

$$
\mathcal{W}_{a} \star_{N} \mathcal{W}_{b}\left(\psi^{\prime}, \bar{\psi}^{\prime}, N\right)=\frac{1}{N} \mathcal{W}_{a} \star_{1} \mathcal{W}_{b}\left(\psi^{\prime}, \bar{\psi}^{\prime}, 1\right)+\frac{N-1}{N} \mathcal{W}_{a}\left(\psi^{\prime}, \bar{\psi}^{\prime}\right) \mathcal{W}_{b}\left(\psi^{\prime}, \bar{\psi}^{\prime}\right)
$$

Introducing the matrix $K$ with

$$
K_{a b}:=\mathcal{W}_{a} \star_{1} \mathcal{W}_{b}-\mathcal{W}_{a} \mathcal{W}_{b}
$$

we can express (80) as

$$
\mathcal{W}_{a} \star_{N} \mathcal{W}_{b}=\frac{1}{N} K_{a b}+\mathcal{W}_{a} \mathcal{W}_{b}
$$

In this form it is apparent that in the graded commutative limit $N \rightarrow \infty$, we recover the graded commutative product of functions $\mathcal{W}_{a}$ and $\mathcal{W}_{b}$.

To construct the $\star$-product of arbitrary functions on $S_{F}^{(2,2)}$ we proceed as follows [9]. Consider first generic operators $F$ and $G$ in the representation $\left(\frac{\hat{N}}{2}\right)_{o s p(2,2)+}$. We expand them in the form

$$
\begin{aligned}
F & =F^{a_{1} a_{2} \cdots a_{N}} \lambda_{a_{1}} \otimes_{G} \cdots \otimes_{G} \lambda_{a_{N}}, \\
G & =G^{b_{1} b_{2} \cdots b_{N}} \lambda_{b_{1}} \otimes_{G} \cdots \otimes_{G} \lambda_{b_{N}}
\end{aligned}
$$


where $F^{a_{1} \cdots a_{i} a_{j} \cdots a_{N}}=(-1)^{\left|a_{i}\right|\left|a_{j}\right|} F^{a_{1} \cdots a_{j} a_{i} \cdots a_{N}},\left|a_{i}\right|(\bmod 2)$ being the degree of the index $a_{i}$. The corresponding functions on $S_{F}^{(2,2)}$ can be read from

$$
<\psi^{\prime}, 1\left|\otimes_{G} \cdots \otimes_{G}<\psi^{\prime}, 1\right|\left\{\begin{array}{l}
F^{a_{1} a_{2} \cdots a_{N}} \lambda_{a_{1}} \otimes_{G} \cdots \otimes_{G} \lambda_{a_{N}} \\
G^{b_{1} b_{2} \cdots b_{N}} \lambda_{b_{1}} \otimes_{G} \cdots \otimes_{G} \lambda_{b_{N}}
\end{array}\left|\psi^{\prime}, 1>\otimes_{G} \cdots \otimes_{G}\right| \psi^{\prime}, 1>\right.
$$

to be

$$
\begin{aligned}
& \mathcal{F}_{N}(\mathcal{W})=F^{a_{1} a_{2} \cdots a_{N}} \mathcal{W}_{a_{1}} \cdots \mathcal{W}_{a_{N}} \\
& \mathcal{G}_{N}(\mathcal{W})=G^{b_{1} b_{2} \cdots b_{N}} \mathcal{W}_{b_{1}} \cdots \mathcal{W}_{b_{N}}
\end{aligned}
$$

In passing from(84) to (85), we have used the fact that $\mid \psi^{\prime}, 1>$ is even.

The $\star$-product of these functions becomes

$$
\begin{aligned}
& \mathcal{F}_{N} \star_{N} \mathcal{G}_{N}(\mathcal{W})=(-1)^{\sum_{j>i}\left|a_{j}\right|\left|b_{i}\right|} F^{a_{1} a_{2} \cdots a_{N}}\left(\mathcal{W}_{a_{1} \star_{1}} \mathcal{W}_{b_{1}}\right) \cdots\left(\mathcal{W}_{a_{N} \star_{1}} \mathcal{W}_{b_{N}}\right) G^{b_{1} b_{2} \cdots b_{N}} \\
& =(-1)^{\sum_{j>i}\left|a_{j}\right|\left|b_{i}\right|} F^{a_{1} a_{2} \cdots a_{N}}\left(\mathcal{W}_{a_{1}} \mathcal{W}_{b_{1}}+K_{a_{1} b_{1}}\right) \cdots\left(\mathcal{W}_{a_{N}} \mathcal{W}_{b_{N}}+K_{a_{N} b_{N}}\right) G^{b_{1} b_{2} \cdots b_{N}}
\end{aligned}
$$

where we have used the formula degree of $\mathcal{W}_{a} \equiv \operatorname{deg} \mathcal{W}_{a}=|a|(\bmod 2)$.

In order to give the $\star$-product in $(\sqrt[86]{ })$ its final form, we proceed as follows. First notice the identity

$$
\begin{aligned}
\mathcal{W}_{a} \mathcal{W}_{b}+K_{a b} & =\mathcal{W}_{a}\left(1+\overleftarrow{\partial}_{\mathcal{W}_{c}} K_{c d} \vec{\partial}_{\mathcal{W}_{d}}\right) \mathcal{W}_{b} \\
& \equiv \mathcal{W}_{a}(1+\bar{\partial} K \vec{\partial}) \mathcal{W}_{b} .
\end{aligned}
$$

Inserting this, R.H.S. of (86) becomes

$$
\begin{aligned}
& (-1)^{\sum_{j>i}\left|a_{j}\right|\left|b_{i}\right|} F^{a_{1} a_{2} \cdots a_{N}}\left(\mathcal{W}_{a_{1}}(1+\bar{\partial} K \vec{\partial}) \mathcal{W}_{b_{1}}\right) \cdots\left(\mathcal{W}_{a_{N}}(1+\bar{\partial} K \vec{\partial}) \mathcal{W}_{b_{N}}\right) G^{b_{1} b_{2} \cdots b_{N}} \\
& \equiv \mathcal{F}_{N}(\mathcal{W})(1+\overleftarrow{\partial} K \vec{\partial})_{11} \cdots(1+\overleftarrow{\partial} K \vec{\partial})_{i i} \cdots(1+\overleftarrow{\partial} K \vec{\partial})_{N N} \mathcal{G}_{N}(\mathcal{W}),
\end{aligned}
$$

where in the second line of (88) we have introduced an auxilary notation whose meaning will now be explained. First we note that we have written $(1+\bar{\partial} K \vec{\partial})_{i i}$ for $1+(\bar{\partial} K \vec{\partial})_{i i}$, purely for notational convenience. Now the meaning of the subscripts in $(\overleftarrow{\partial} K \vec{\partial})_{i i}$ is as follows: Consider the expansion of $\mathcal{F}_{N}$ and $\mathcal{G}_{N}$ given in (85). There are $N$ slots in both $\mathcal{F}_{N}$ and $\mathcal{G}_{N}$ each occupied by some $\mathcal{W}_{a}$ and $\mathcal{W}_{b}$ respectively. The first subscript $i$ means that $\delta$ in $(\delta K \vec{\partial})_{i i}$ acts only on the $\mathcal{W}_{a}$ in the $i^{\text {th }}$ slot (counting from left) in the expansion of $\mathcal{F}_{N}$ in $(85)$, whereas the second subscript $i$ means that $\vec{\partial}$ in $(\overleftarrow{\partial} K \vec{\partial})_{i i}$ acts only on the $\mathcal{W}_{b}$ in the $i^{\text {th }}$ slot(counting from left) in the expansion of $\mathcal{G}_{N}$ in (85). We also note the following:

$$
\begin{aligned}
& \text { i) } \mathcal{F}_{N}(\mathcal{W})(\overleftarrow{\partial} K \vec{\partial})_{i i} \mathcal{G}_{N}(\mathcal{W})=\mathcal{F}_{N}(\mathcal{W})(\overleftarrow{\partial} K \vec{\partial})_{j j} \mathcal{G}_{N}(\mathcal{W}) \\
& \text { ii) } \mathcal{F}_{N}(\mathcal{W})\left(\overleftarrow{\partial}_{\mathcal{W}_{a_{j}}}\right)_{i}\left(\overleftarrow{\partial}_{\mathcal{W}_{a_{k}}}\right)_{i}=\left(\vec{\partial}_{\mathcal{W}_{b_{j}}}\right)_{i}\left(\vec{\partial}_{\mathcal{W}_{b_{k}}}\right)_{i} \mathcal{G}_{N}(\mathcal{W})=0
\end{aligned}
$$

Here $\mathcal{F}_{N}(\mathcal{W})\left(\overleftarrow{\partial}_{\mathcal{W}_{a_{m}}}\right)_{i}\left(\left(\vec{\partial}_{\mathcal{W}_{b_{m}}}\right)_{i} \mathcal{G}_{N}(\mathcal{W})\right)$ means that we apply the derivative $\overleftarrow{\partial}_{\mathcal{W}_{a_{m}}}\left(\vec{\partial}_{\mathcal{W}_{b_{m}}}\right)$ only on the $i^{\text {th }}$ slot in the expansion of $\mathcal{F}_{N}(\mathcal{W})\left(\mathcal{G}_{N}(\mathcal{W})\right)$ in the sense explained above. It takes a simple relabeling of the indices and the use of symmetries of $\mathcal{F}_{N}(\mathcal{W})$ and $\mathcal{G}_{N}(\mathcal{W})$ to prove $i$ ) while $\left.i i\right)$ is obvious by inspection. 
We can write (88) as

$$
\begin{aligned}
& \mathcal{F}_{N{ }_{N}} \mathcal{G}_{N}(\mathcal{W})=\mathcal{F}_{N} \mathcal{G}_{N}(\mathcal{W}) \\
& +\sum_{m=1}^{N} \frac{N !}{(N-m) ! m !} \mathcal{F}_{N}(\mathcal{W})\left((\overleftarrow{\partial} K \vec{\partial})_{11} \cdots(\bar{\partial} K \vec{\partial})_{i i} \cdots(\bar{\partial} K \vec{\partial})_{m m}\right) \mathcal{G}_{N}(\mathcal{W})
\end{aligned}
$$

Now observe the following identities:

$$
\begin{aligned}
& \mathcal{F}_{N}(\mathcal{W})\left(\overleftarrow{\partial}_{\mathcal{W}_{a_{1}}}\right)_{1} \cdots\left(\overleftarrow{\partial}_{\mathcal{W}_{a_{1}}}\right)_{i} \cdots\left(\overleftarrow{\partial}_{\mathcal{W}_{a_{m}}}\right)_{m}=\frac{(N-m) !}{N !} \mathcal{F}_{N}(\mathcal{W}) \overleftarrow{\partial}_{\mathcal{W}_{a_{1}}} \cdots \overleftarrow{\partial}_{\mathcal{W}_{a_{i}}} \cdots \overleftarrow{\partial}_{\mathcal{W}_{a_{m}}}, \\
& \left(\vec{\partial}_{\mathcal{W}_{b_{1}}}\right)_{1} \cdots\left(\vec{\partial}_{\mathcal{W}_{b_{m}}}\right)_{i} \cdots\left(\vec{\partial}_{\mathcal{W}_{b_{m}}}\right)_{m} \mathcal{G}_{N}(\mathcal{W})=\frac{(N-m) !}{N !} \vec{\partial}_{\mathcal{W}_{b_{1}}} \cdots \vec{\partial}_{\mathcal{W}_{b_{i}}} \cdots \vec{\partial}_{\mathcal{W}_{b_{m}}} \mathcal{G}_{N}(\mathcal{W})
\end{aligned}
$$

To facilitate the use of (91) in (90) we define the ordering $\vdots \ldots \vdots$ in which $\overleftarrow{\partial}_{\mathcal{W}_{a_{i}}}\left(\vec{\partial}_{\mathcal{W}_{b_{i}}}\right)$ are moved to the left (right) extreme and they act on everything to their left (right) :

$$
\begin{aligned}
& \vdots(1+\overleftarrow{\partial} K \vec{\partial})^{N} \vdots=1+\sum_{m=1}^{N} \frac{N !}{(N-m) ! m !} \vdots \underbrace{(\overleftarrow{\partial} K \vec{\partial}) \cdots(\overleftarrow{\partial} K \vec{\partial})}_{m \text { factors }} \vdots \\
& \equiv 1+\sum_{m=1}^{N} \frac{N !}{(N-m) ! m !}(-1)^{\Delta} \overleftarrow{\partial}_{\mathcal{W}_{a_{1}}} \cdots \overleftarrow{\partial}_{\mathcal{W}_{a_{m}}} K_{a_{1} b_{1}} \cdots K_{a_{m} b_{m}} \vec{\partial}_{\mathcal{W}_{b_{1}}} \cdots \vec{\partial}_{\mathcal{W}_{b_{m}}} .
\end{aligned}
$$

where $\Delta=\sum_{j>i}^{m}\left(\left|a_{j}\right|\left|a_{i}\right|+\left|a_{j}\right|\left|b_{i}\right|+\left|b_{j}\right|\left|b_{i}\right|\right)(\bmod 2)$ is the overall degree due to moving the derivatives to their positions in (92). Using (91) and (92) in (90) we write our $\star$-product in its final form

$$
\mathcal{F}_{N} \star_{N} \mathcal{G}_{N}(\mathcal{W})=\mathcal{F}_{N} \mathcal{G}_{N}(\mathcal{W})+\sum_{m=1}^{N} \frac{(N-m) !}{N ! m !} \mathcal{F}_{N}(\mathcal{W}) \vdots \underbrace{(\overleftarrow{\partial} K \vec{\partial}) \cdots(\overleftarrow{\partial} K \vec{\partial})}_{m \text { factors }} \vdots \mathcal{G}_{N}(\mathcal{W})
$$

It depends on the ordering introduced in (92) and not on the auxilary notation of (88) and (91). From this formula it is apparent that, in the graded commutative limit $(N \rightarrow \infty)$, we get back the ordinary pointwise multiplication $\mathcal{F}_{N} \mathcal{G}_{N}(\mathcal{W})$.

\section{$5.2 \star$-Product on Fuzzy "Sections of Bundles"}

In this subsection we extend the $\star$-product found above to functions obtained from matrix elements of annihilation-creation operators between vectors of different $N$, using the ideas of [1]. Linear operators between vector spaces of different $N$ correspond to sections of bundles [13, 8, 5] so that in this manner, we extend our supersymmetric $\star$-product to sections of bundles. The results below also provide an alternative way to compute the $\star$-products in (80) and (93).

First note that with $\left[\Psi_{\mu}, \Psi_{\nu}^{\dagger}\right\}=\delta_{\mu \nu}$, we have

$$
\begin{aligned}
& \Psi_{\mu}\left|\psi, N>=\sqrt{N} \frac{\psi_{\mu}}{|\psi|}\right| \psi, N-1> \\
& <\psi, N\left|\Psi_{\mu}^{\dagger}=<\psi, N-1\right| \sqrt{N} \frac{\bar{\psi}_{\mu}}{|\psi|} .
\end{aligned}
$$


We now let $\psi^{\prime}=\psi /|\psi|$ as before and define

$$
\begin{aligned}
S_{\mu} & :=\Psi_{\mu} \frac{1}{\sqrt{N}}=\frac{1}{\sqrt{N+1}} \Psi_{\mu}, \\
S_{\mu}^{\dagger} & :=\frac{1}{\sqrt{N}} \Psi_{\mu}^{\dagger}=\Psi_{\mu}^{\dagger} \frac{1}{\sqrt{N+1}}
\end{aligned}
$$

where $N=\Psi_{\mu}^{\dagger} \Psi_{\mu}$ is the number operator. Then

$$
\begin{aligned}
& S_{\mu}\left|\psi^{\prime}, N>=\psi_{\mu}^{\prime}\right| \psi^{\prime}, N-1>, \\
& <\psi^{\prime}, N\left|S_{\mu}^{\dagger}=<\psi^{\prime}, N-1\right| \bar{\psi}_{\mu}{ }^{\prime} .
\end{aligned}
$$

Furthermore, we have that $\left[S_{\mu}, S_{\nu}\right\}=\left[S_{\mu}^{\dagger}, S_{\nu}^{\dagger}\right\}=0$ while after a small calculation we get

$$
\left[S_{\mu}, S_{\nu}^{\dagger}\right\}=\frac{1}{N+1}\left(\delta_{\mu \nu}-(-1)^{\left|S_{\mu}\right|\left|S_{\nu}\right|} S_{\nu}^{\dagger} S_{\mu}\right)
$$

where $\left|S_{\mu}\right|$ denotes the degree of $S_{\mu}$. Using (99), we also get

$$
\begin{aligned}
& <\psi^{\prime}, N-1\left|S_{\mu}\right| \psi^{\prime}, N>=\psi_{\mu}^{\prime} \\
& <\psi^{\prime}, N\left|S_{\mu}^{\dagger}\right| \psi^{\prime}, N-1>=\bar{\psi}_{\mu}^{\prime} .
\end{aligned}
$$

Thus, the $\star$-product of $\psi^{\prime}$ with $\bar{\psi}^{\prime}$ is given by

$$
\begin{aligned}
\psi_{\mu}^{\prime} \star \bar{\psi}_{\nu}^{\prime} & =<\psi^{\prime}, N\left|S_{\mu} S_{\nu}^{\dagger}\right| \psi^{\prime}, N> \\
& =<\psi^{\prime}, N\left|(-1)^{\left|S_{\mu}\right|\left|S_{\nu}\right|} \frac{N}{N+1} S_{\nu}^{\dagger} S_{\mu}+\frac{1}{N+1} \delta_{\mu \nu}\right| \psi^{\prime}, N> \\
& =\frac{N}{N+1} \psi_{\mu}^{\prime} \bar{\psi}_{\nu}^{\prime}+\frac{1}{N+1} \delta_{\mu \nu} .
\end{aligned}
$$

Here we have used (100) and the fact that $\psi_{\mu}^{\prime} \bar{\psi}_{\nu}^{\prime}=(-1)^{\left|S_{\mu}\right|\left|S_{\nu}\right|} \bar{\psi}_{\nu}^{\prime} \psi_{\mu}^{\prime}$ to get rid of $(-1)^{\left|S_{\mu}\right|\left|S_{\nu}\right|}$. Rearreaging the last result we can write

$$
\begin{aligned}
\psi_{\mu}^{\prime} \star \bar{\psi}_{\nu}^{\prime} & =\frac{1}{N+1} \Omega_{\mu \nu}+\psi_{\mu}^{\prime} \bar{\psi}_{\nu}^{\prime}, \\
\Omega_{\mu \nu} & \equiv \delta_{\mu \nu}-\psi_{\mu}^{\prime} \bar{\psi}_{\nu}^{\prime} .
\end{aligned}
$$

As an easy check of our results, we can compute $\mathcal{W}_{a} \star_{N} \mathcal{W}_{b}$, using the method above. First note that

$$
\mathcal{W}_{a}=\bar{\psi}^{\prime} \Lambda_{a} \psi^{\prime}=<\psi^{\prime}, N\left|S^{\dagger} \Lambda_{a} S\right| \psi^{\prime}, N>
$$

Hence

$$
\begin{aligned}
\mathcal{W}_{a} \star_{N} \mathcal{W}_{b} & =<\psi^{\prime}, N\left|S_{\mu}^{\dagger}\left(\Lambda_{a}\right)_{\mu \nu} S_{\nu} S_{\alpha}^{\dagger}\left(\Lambda_{b}\right)_{\alpha \beta} S_{\beta}\right| \psi^{\prime}, N> \\
& =\bar{\psi}_{\mu}^{\prime}\left(\Lambda_{a}\right)_{\mu \nu}\left(\frac{1}{N} \Omega_{\nu \alpha}+\psi_{\nu}^{\prime} \bar{\psi}_{\alpha}^{\prime}\right)\left(\Lambda_{b}\right)_{\alpha \beta} \psi_{\beta}^{\prime} \\
& =\bar{\psi}_{\mu}^{\prime}\left(\Lambda_{a}\right)_{\mu \nu}\left(\frac{1}{N} \delta_{\nu \alpha}+\frac{N-1}{N} \psi_{\nu}^{\prime} \bar{\psi}_{\alpha}^{\prime}\right)\left(\Lambda_{b}\right)_{\alpha \beta} \psi_{\beta}^{\prime} \\
& =\frac{1}{N} \mathcal{W}_{a} \star_{1} \mathcal{W}_{b}+\frac{N-1}{N} \mathcal{W}_{a} \mathcal{W}_{b}
\end{aligned}
$$


which is (80).

Comparing the second line of the last equation with (82) we get the important result ff

$$
\begin{aligned}
K_{a b} & =\left(\mathcal{W}_{a} \overleftarrow{\partial}_{\mu}\right) \Omega_{\mu \nu}\left(\vec{\partial}_{\nu} \mathcal{W}_{b}\right) \\
& \equiv \mathcal{W}_{a} \bar{\partial} \Omega \vec{\partial} \mathcal{W}_{b}
\end{aligned}
$$

where $\overleftarrow{\partial} \Omega \vec{\partial} \equiv \overleftarrow{\partial}_{\mu} \Omega_{\mu \nu} \vec{\partial}$ and $\partial_{\mu}=\frac{\partial}{\partial \psi_{\mu}^{\prime}}$.

We would like to note that this result can be used to write (93) in terms of $\bar{\partial} \Omega \vec{\partial}$. To this end we write (86) as

$$
(-1)^{\sum_{j>i}\left|a_{j}\right|\left|b_{i}\right|} F^{a_{1} a_{2} \cdots a_{N}}\left(\mathcal{W}_{a_{1}}(1+\bar{\partial} \Omega \vec{\partial}) \mathcal{W}_{b_{1}}\right) \cdots\left(\mathcal{W}_{a_{N}}(1+\bar{\partial} \Omega \vec{\partial}) \mathcal{W}_{b_{N}}\right) G^{b_{1} b_{2} \cdots b_{N}}
$$

Carrying out a calculation similar to the one given in (88) through (93), one finally finds

$$
\mathcal{F}_{N} \star_{N} \mathcal{G}_{N}(\mathcal{W})=\mathcal{F}_{N} \mathcal{G}_{N}(\mathcal{W})+\sum_{m=1}^{N} \frac{(N-m) !}{N ! m !} \mathcal{F}_{N}(\mathcal{W}) \vdots \underbrace{(\overleftarrow{\partial} \Omega \overrightarrow{\bar{\partial}}) \cdots(\bar{\partial} \Omega \overrightarrow{\bar{\partial}})}_{m \text { factors }} \vdots \mathcal{G}_{N}(\mathcal{W})
$$

where now $\vdots \ldots \vdots$ takes $\bar{\partial}$ and $\vec{\partial}$ to the left and right extreme respectively. (When $\bar{\partial}$ 's and $\vec{\partial}$ 's are moved in this fashion, the phases coming from the graded commutators should be included just as for (93)).

It can be explicitly shown that $\Omega=\left(\Omega_{\mu \nu}\right)$ is a projector, i.e.,

$$
\Omega^{2}=\Omega \quad \text { and } \quad \Omega^{\ddagger}=\Omega .
$$

Due to (107), the last equation implies similar properties for 9

$$
\mathcal{K}_{a b} \equiv\left(K S^{-1}\right)_{a b}
$$

which we discuss next.

\section{More on the Properties of $\mathcal{K}_{a b}$}

A closer look at the properties of $\mathcal{K}_{a b} \equiv\left(K S^{-1}\right)_{a b}$, where

$$
\begin{aligned}
K_{a b}(\psi) & =\mathcal{W}_{a} \star_{1} \mathcal{W}_{b}(\psi)-\mathcal{W}_{a}(\psi) \mathcal{W}_{b}(\psi) \\
& =<\psi, 1\left|\lambda_{a} \lambda_{b}\right| \psi, 1>-<\psi, 1\left|\lambda_{a}\right| \psi, 1><\psi, 1\left|\lambda_{b}\right| \psi, 1>
\end{aligned}
$$

will give us more insight on the structure of the $\star$-product found in the previous section. First note that $\mathcal{K}_{a b}$ depends on both $\psi$ and $\bar{\psi}$. We denote this dependence by $\mathcal{K}_{a b}(\psi)$ for short, omitting to write the $\bar{\psi}$ dependence. Now we would like to show that the matrix $\mathcal{K}(\psi)=\left(\mathcal{K}_{a b}(\psi)\right)$ is a projector.

\footnotetext{
${ }^{4}$ We thank Peter Prešnajder for a discussion leading to (107)

${ }^{5}$ Following the conventions of [12], we consider all the indices down through out this paper. In what follows the relevant object under investigation is $\mathcal{K}_{a b}$ corresponding to $K_{a}{ }^{b}$ in a notation where indices are raised and lowered by the metric. To stick with the conventions of [12], we write (111) and proceed accordingly.
} 
We first recall that in $\left(\frac{\hat{1}}{2}\right)_{\operatorname{osp}(2,2)+}$, representation of $\operatorname{osp}(2,2)$ the highest and lowest weight states are given by

$$
\mid J, J_{3}>= \begin{cases}\mid 1 / 2,1 / 2>=\text { highest weight state } \\ \mid 1 / 2,-1 / 2>=\text { lowest weight state }\end{cases}
$$

We note that, starting from the lowest weight state $\left|1 / 2,-1 / 2>=\Psi_{2}^{\dagger}\right| 0>$, one could construct another supercoherent state, expressed by a formula similar to (43). Now consider the following fiducial points for $\mathcal{W}(\psi)$ at $\psi=\psi^{0}=(1,0,0)$ obtained from computing $\mathcal{W}_{a}\left(\psi^{0}\right)$ in the supercoherent states induced from the states given in (113):

$$
\mathcal{W}^{ \pm}\left(\psi^{0}\right)=\left(\mathcal{W}_{1}\left(\psi^{0}\right) \cdots \mathcal{W}_{8}\left(\psi^{0}\right)\right)=(0,0, \pm 1 / 2,0,0,0,0,1)
$$

In $(114)+(-)$ correponds to upper(lower) entries in (113) and the calculation is done using (76).

Although not essential in what follows, we remark that $\mathcal{W}^{-}(\psi=(1,0,0))=\mathcal{W}^{+}(\psi=(0,1,0))$, that is,

$$
\mathcal{W}_{a}^{-}\left(\psi^{0}\right)=\mathcal{W}_{b}^{+}\left(\psi^{0}\right)\left(A d e^{i \pi \Lambda_{2}^{\left(\frac{1}{2}\right)}}\right)_{b a}
$$

Note that all other points in $S_{F}^{(2,2)}$ can be obtained from $\mathcal{W}^{ \pm}\left(\psi^{0}\right)$ by the adjoint action of the group, i.e.,

$$
\mathcal{W}_{a}^{ \pm}(\psi)=\mathcal{W}_{b}^{ \pm}\left(\psi^{0}\right)\left(A d g^{-1}\right)_{b a}
$$

where $\psi=g \psi^{0}$.

We define $\mathcal{K}^{ \pm}\left(\psi^{0}\right)$ using $\mathcal{W}^{ \pm}\left(\psi^{0}\right)$ for $\mathcal{W}$, (111) and (112). The matrices $\mathcal{K}^{ \pm}\left(\psi^{0}\right)$ when computed at the fiducial points (using for instance (22, 79, 81)) have the block diagonal forms

$$
\mathcal{K}^{ \pm}\left(\psi^{0}\right)=\left(\mathcal{K}_{a b}^{ \pm}\left(\psi^{0}\right)\right)=\left(\begin{array}{ccc}
\left(\frac{1}{2} \delta_{i j} \pm \frac{i}{2} \epsilon_{i j 3}-2 \mathcal{W}_{i}^{ \pm}\left(\psi^{0}\right)\left(\mathcal{W}_{j}^{ \pm}\left(\psi^{0}\right)\right)\right)_{3 \times 3} & & \\
& \left(\Sigma_{\alpha \beta}^{ \pm}\right)_{4 \times 4} & \\
& & 0
\end{array}\right)_{8 \times 8}
$$

with

$$
\Sigma^{ \pm}=\left(\Sigma_{\alpha \beta}^{ \pm}\right)=\frac{1}{4}\left(\begin{array}{cc}
1 \pm \sigma_{3} & -\left(1 \pm \sigma_{3}\right) \\
-\left(1 \pm \sigma_{3}\right) & 1 \pm \sigma_{3}
\end{array}\right)
$$

where the upper (lower) sign stands for the upper (lower) sign in $\mathcal{W}^{ \pm}\left(\psi^{0}\right)$. The matrices $\mathcal{K}^{ \pm}\left(\psi^{0}\right)$ have only even components and do not mix the 1,2,3,8 and 4,5,6,7 entries of a vector. So its grade adjoint is its ordinary adjoint $\dagger$. Now from (117) it is straightforward to check the relations

$$
\begin{gathered}
\left(\mathcal{K}^{ \pm}\left(\psi^{0}\right)\right)^{2}=\mathcal{K}^{ \pm}\left(\psi^{0}\right) \\
\left(\mathcal{K}^{ \pm}\left(\psi^{0}\right)\right)^{\ddagger}=\mathcal{K}^{ \pm}\left(\psi^{0}\right) \\
\mathcal{K}^{+}\left(\psi^{0}\right) \mathcal{K}^{-}\left(\psi^{0}\right)=0
\end{gathered}
$$


which show that $\mathcal{K}^{ \pm}\left(\psi^{0}\right)$ are orthogonal projectors. By the adjoint action of the group, we have

$$
\mathcal{K}_{a b}^{ \pm}(\psi)=\left((A d g)^{T}\right)_{a d}^{-1} \mathcal{K}_{d e}^{ \pm}\left(\psi^{0}\right)(A d g)_{e b}^{T},
$$

with $T$ denoting transpose, implying that $\mathcal{K}^{ \pm}(\psi)$ are projectors for all $g \in O S p(2,2)$.

We further observe that a super-analogue $\mathcal{J}$ of the complex structure can be defined over the supersphere. To show this, following [9], we first observe that the projective module for "sections of the supertangent bundle" over $S^{(2,2)}$ is $\mathcal{P} \mathcal{A}^{8}$, where $\mathcal{A}$ is the algebra of superfunctions over $S^{(2,2)}, \mathcal{A}^{8}=\mathcal{A} \otimes_{\mathbb{C}} \mathbb{C}^{8}$ and $\mathcal{P}(\psi)=\mathcal{K}^{+}(\psi)+\mathcal{K}^{-}(\psi)$ is a projector. (For details, see [9].) The super-complex structure then is given by the matrix $\mathcal{J}$ with elements

$$
\mathcal{J}_{a b}(\psi)=i\left(\mathcal{K}^{+}-\mathcal{K}^{-}\right)_{a b}(\psi) .
$$

It acts on $\mathcal{P} \mathcal{A}^{8}$. Since

$$
\left.\mathcal{J}^{2}(\psi)\right|_{\mathcal{P A}^{8}}=-\left.\mathcal{P}(\psi)\right|_{\mathcal{P A}^{8}}=-\left.\mathbf{1}\right|_{\mathcal{P} \mathcal{A}^{8}}
$$

$\left(\left.\delta\right|_{\varepsilon}\right.$ denoting the restriction of $\delta$ to $\left.\varepsilon\right)$, it defines a super complex structure. Furthermore, due to the relation

$$
\left.\mathcal{J}\right|_{\mathcal{K}^{ \pm} \mathcal{A}^{8}}= \pm\left. i\right|_{\mathcal{K}^{ \pm} \mathcal{A}^{8}}
$$

$\mathcal{K}^{ \pm} \mathcal{A}^{8}$ give the "holomorphic" and "anti-holomorphic" parts of $\mathcal{P} \mathcal{A}^{8}$.

Finally we also have

$$
\mathcal{K}^{ \pm}(\psi)=\frac{1}{2}\left(-\mathcal{J}^{2} \mp i \mathcal{J}\right)(\psi)
$$

\section{Discussion and Conclusions}

In this article we have constructed the $\star$-product of functions on $S_{F}^{(2,2)}$. Our central result is given in (93). A consequence of (80) is the graded commutator of the $\star$-product

$$
\left[\mathcal{W}_{a}, \mathcal{W}_{b}\right\}_{\star_{N}}=\frac{i}{N} f_{a b c} \mathcal{W}_{c}
$$

which generalizes a familiar result for the usual $\star$-products. A special case of our result for the $\star$-product follows if we restrict ourselves to the even subspace $S_{F}^{2}$ of $S_{F}^{(2,2)}$, namely the fuzzy sphere. In this case, we get from (80) and (93),

$$
\begin{gathered}
\mathcal{F}_{N} \star_{N} \mathcal{G}_{N}(\mathcal{W})=\mathcal{F}_{N} \mathcal{G}_{N}(\mathcal{W})+ \\
\sum_{m=1}^{N} \frac{(N-m) !}{N ! m !}\left(\partial_{a_{1}} \cdots \partial_{a_{m}} \mathcal{F}_{N}(\mathcal{W})\right) K_{a_{1} b_{1}} \cdots K_{a_{m} b_{m}}\left(\partial_{b_{1}} \cdots \partial_{b_{m}} \mathcal{G}_{N}(\mathcal{W})\right), \\
\partial_{a_{i}} \equiv \partial_{\mathcal{W}_{a_{i}}}, \quad \partial_{b_{j}} \equiv \partial_{\mathcal{W}_{b_{j}}},
\end{gathered}
$$

which is the formula given in $[8,9]$. 
There have been developments in writing sigma models in $S_{F}^{2}$ using Bott projectors [7]. It appears that the projector $\Omega$ introduced in (107) is the supersymmetric version of Bott projector

(for Chern class 1) and can be the starting point for constructing sigma models on $S_{F}^{(2,2)}$. We will develop this idea in another article.

\section{Acknowledgements}

The authors are indebted to Denjoe O'Connor and Peter Prešnajder for useful discussions and Peter Prešnajder for detailed comments on paper. E.R. would like to thank Department of Physics of Syracuse University for hospitality during his stay in Syracuse and acknowledges support from a CONACyT post-doctoral fellowship. The work of A.P.B and S.K. was supported in part by DOE and NSF under contract numbers DE-FG02-85ER40231 and INT9908763 respectively.

\section{References}

[1] D. O'Connor and P. Prešnajder, private communication.

[2] J. Madore, An Introduction to Noncommutative Differential Geometry and its Physical Applications, Cambridge University Press, Cambridge (1995); J. Madore, Class. Quant. Grav. 9 (1991) 69.

[3] B. Ydri, Ph.D. Thesis Syracuse University, Syracuse NY (2001) and hep-th/0110006.

[4] H. Grosse, C. Klimčik, P. Prešnajder, Int. J. Theor .Phys. 35 (1996) 231 and hep-th/9505175; B. Ydri Phy.Rev.D 63 (2001) 25004.

[5] A. P. Balachandran and S. Vaidya Int. J. Mod. Phy. A16 (2001) 17 and hep-th/9910129; S. Baez, A. P. Balachandran, S. Vaidya, and B. Ydri, Commun. Math. Phys. 208 (2000) 787 and hep-th/9811169.

[6] A. P. Balachandran, T. R. Govindarajan and B.Ydri Mod. Phys. Lett.A15 (2000) 1279 and hep-th/9911087; A. P. Balachandran, T. R. Govindarajan and B.Ydri hep-th/0006216.

[7] A. P. Balachandran and G.Immirzi, in preparation, see also Baez et. al. in [5].

[8] P. Prešnajder, J. Math. Phys. 41 (2000) 2789-2804 and hep-th/9912050.

[9] A. P. Balachandran, B. Dolan, J. Lee, X. Martin and D. O'Connor, Fuzzy Complex Projective Spaces and their Star-Products, hep-th/0107099 and J. Geom. and Phys. (in press).

[10] B. Dolan and O. Jahn, Fuzzy Complex Grassmannian Spaces and their Star Products, hepth/0111020.

[11] A. M. Perelomov Generalized Coherent States and their Applications, Springer-Verlag (1986); M. Bordemann, M. Brischle, C. Emmrich and S. Waldmann, J. Math. Phys. 37 (1996) 6311; M. Bordemann, M. Brischle, C. Emmrich and S. Waldmann, Lett. Math. Phys. 36 (1996) 357; S. Waldmann, Lett. Math. Phys. 44 (1998) 331. 
[12] H. Grosse, C. Klimčik, P. Prešnajder, N=2 Superalgebra and Non-Commutative Geometry, hep-th/9603071; H. Grosse, C. Klimcik, P. Presnajder, Field Theory on a Supersymmetric Lattice,Commun. Math. Phys. 185 (1997) 155-175 and hep-th/9507074.

[13] H. Grosse, C. Klimčik, P. Prešnajder, Commun. Math. Phys. 178 (1996) 507-526 and hepth/9510083; H. Grosse, P. Prešnajder, Lett. Math. Phys. 46 (1998) 61-69.

[14] H. Grosse, G. Reiter, The Fuzzy Supersphere, J. Geom. and Phys. 28 (1998) 349-383 and math-ph/9804013.

[15] B. Dewitt, Supermanifolds, Cambridge University Press, Cambridge (1985); M. Scheunert, The Theory of Lie Superalgebras, Springer-Verlag, Berlin (1979).

[16] J. F. Cornwell, Group Theory in Physics Vol. III, Academic Press, San Diego (1989).

[17] A. Pais and V. Rittenberg, J. Math. Phys. 16 (1975) 2062.

[18] M. Scheunert, W. Nahm and V. Rittenberg, J. Math. Phys. 18 (1977) 146.

[19] M. Scheunert, W. Nahm and V. Rittenberg, J. Math. Phys. 18 (1977) 154.

[20] C. Klimčik, Commun. Math. Phys. 206 (1999) 567-586 and hep-th/9903112.

[21] M. Chaichian, D. Ellinias, P. Prešnajder, J. Math. Phys. 32 (1991) 3381.

[22] A. El Gradechi and L. M. Nieto, Commun. Math. Phys. 175 (1996) 521 and hep-th/9403109; A. El Gradechi, J. Math. Phys. 34 (1993) 5951 and hep-th/9301132.

[23] J. R. Klauder and B. S. Skagerstam, Coherent States: Applications in Physics and Mathematical Physics, World Scientific (1985).

[24] A. P. Balachandran, G.Marmo, B. S. Skagerstam and A. Stern, Nucl. Phys. B164 (1980) 427; G. Landi and G. Marmo, Phy. Lett. B193 (1987) 61-66.

[25] G. Landi, Projective Modules of Finite Type over the Supersphere $S^{2,2}$, Differ. Geom. Appl. 14 (2001) 95-111 and math-ph/9907020.

[26] F. A. Berezin, Introduction to Superanalysis, D.Reidel Publishing Company, Dordrecht, Holland (1987). 